\title{
BASELINE ERROR ANALYSIS AND EXPERIMENTAL VALIDATION FOR HEIGHT MEASUREMENT OF FORMATION INSAR SATELLITE
}

\author{
Xiaoming Gao ${ }^{1,2,3}$, Tao $\mathrm{Li}^{1,}{ }^{*}$, Xiang Zhang ${ }^{1}$, Xiaomeng Geng ${ }^{1}$ \\ ${ }^{1}$ Satellite Surveying and Mapping Application Center, National Administration of Surveying, Mapping and Geo-information, \\ Beijing, China - (gaoxm, lit, zhangx, gengxm)@sasmac.cn \\ ${ }^{2}$ Key Laboratory of Satellite Mapping Technology and Application, NASG, Beijing, China \\ ${ }^{3}$ Jiangsu Center for Collaborative Innovation in Geographical Information Resource Development and \\ Application, Nanjing, China
}

Commission III, WG III/3

KEY WORDS: InSAR, Height Measurement, Baseline Error, DEM, Simulation Validation, SRTM, TerraSAR-X/TanDEM-X

\begin{abstract}
:
In this paper, we proposed the stochastic model of InSAR height measurement by considering the interferometric geometry of InSAR height measurement. The model directly described the relationship between baseline error and height measurement error. Then the simulation analysis in combination with TanDEM-X parameters was implemented to quantitatively evaluate the influence of baseline error to height measurement. Furthermore, the whole emulation validation of InSAR stochastic model was performed on the basis of SRTM DEM and TanDEM-X parameters. The spatial distribution characteristics and error propagation rule of InSAR height measurement were fully evaluated.
\end{abstract}

\section{INTRODUCTION}

Formation flying satellites equipped with synthetic aperture radar (SAR) could provide significant science opportunities, such as generating accurate digital elevation model from Interferometric SAR (InSAR) (Krieger et al., 2007, 2010; Pitz and Miller, 2010). Compared with the single SAR satellite system, the performance of the formation SAR satellites can be greatly enhanced. Nowadays, satellite formation flying has become the focus of remote sensing and space technology.

In order to achieve the advanced goal of InSAR mission, the accurate determination of interferometric baseline is a fundamental issue (Wang, 2005). TanDEM-X is a SAR mission using two radar satellites flying in close formation. The primary goal is the generation of a high-precision global DEM according to high resolution terrain information HRTI-3 standard (Krieger et al., 2007; Zink et al., 2006; Werninghaus and Buckreuss, 2010). The generation of accurate InSARderived DEM requires a precise knowledge of the interferometric baseline with an accuracy of $1 \mathrm{~mm}$ (Wermuth et al., 2011). Therefore high-precision determination of intersatellite interferometric baseline is a prerequisite for formation InSAR system.

The spaceborne dual-frequency GPS measurement scheme is widely applied for inter-satellite baseline determination (Kroes et al., 2005; Montenbruck et al., 2007). This scheme for spatial baseline determination consists of two steps. Firstly, the relative position of two formation satellites is determined by dualfrequency GPS measurement, and then spatial baseline is transformed from inter-satellite relative position. The relative position is the vector that links the mass centres of two formation satellites.

In this research, the impact of baseline error to InSAR height measurement was comprehensively discussed, and simulation validation was implemented to evaluate the spatial distribution characteristics of the height error induced by baseline error. This paper starts with a description of InSAR height measurement observation geometry. The characteristic of the baseline error is analysed, and the baseline error model is constructed on the basis of the first derivative model. In combination with the TanDEM-X parameters, the theoretical relationship between baseline error and height measurement error was quantitatively evaluated. Furthermore, based on the SRTM DEM and TanDEM-X parameters, the simulation validation of the baseline error model was performed to assess the spatial distribution of height error. In conclusion, according to the baseline error evaluation and validation, high precision InSAR spatial baseline determination should be achieved for InSAR height measurement.

The paper is organized as follows. The methodologies of baseline error analysis and simulation strategies are presented in section 2. An overview of the study area and description of the data set is presented in section 3. The results and discussions in section 4 are divided into two parts associated with 1) Point scale evaluation based on the physical model and 2) Spatial distribution validation of the baseline error to height measurement. Finally, the conclusions are presented in section 5.

\section{METHODOLOGY}

\subsection{Baseline error model of InSAR}

The observation geometry of the distributed InSAR measurement is shown as Figure 1. $S_{1}$ and $S_{2}$ are the antenna phase centres corresponding to the master and slave images, respectively. The spatial baseline is generated due to the position difference between $S_{1}$ and $S_{2}$, which is denoted as $B$. $\alpha$ represents the angle between the baseline and horizontal

*Corresponding author 
direction. $\theta$ is the radar incidence angle. $B_{\perp}$ denotes the perpendicular baseline. $r$ and $r+\Delta r$ are the slant range between the master and slave antenna phase centres to target point, respectively. $H$ is the satellite altitude, $R_{\mathrm{H}}$ and $R_{\mathrm{h}}$ are the distance from satellite and ground point to earth centre, respectively. $R$ e denotes the earth radius, and $h$ is the height of the ground point.

Figure 1 shows the observation geometry of the InSAR measurement. According to the cosine law, the geometry representation can be obtained in $S_{1} O P$ triangle.

$$
\left(h+R_{e}\right)^{2}=R_{\mathrm{H}}^{2}+r^{2}-2 r R_{H} \cos \theta
$$

Thus, $h$ can be represented as:

$$
h=\sqrt{R_{H}^{2}+r^{2}-2 r R_{H} \cos \theta}-R_{e}
$$

where

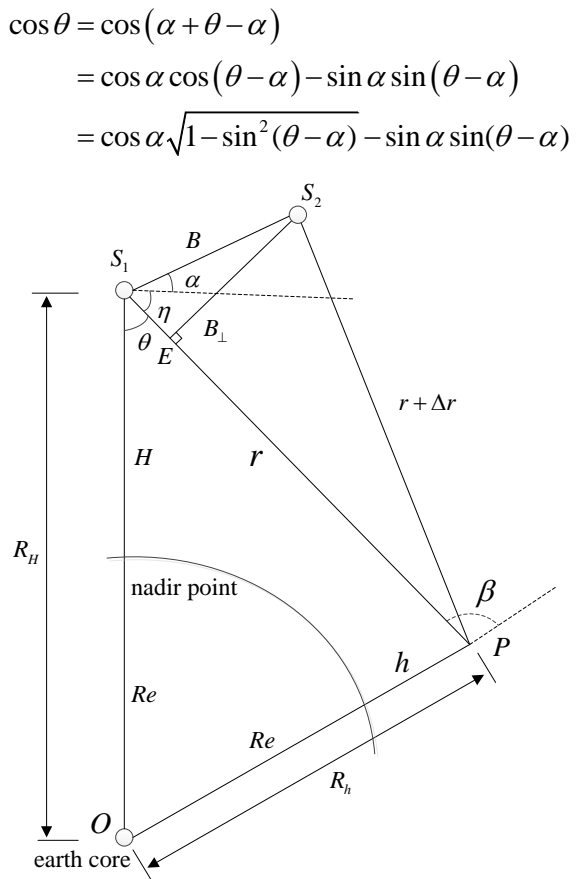

In $S_{1} P S_{2}$ triangle, the following geometry relationship can be derived on the basis of cosine law.

$$
\cos (\eta+\alpha)=\frac{B^{2}+r^{2}-(r+\Delta r)^{2}}{2 B r}
$$

where

$$
\Delta r=\frac{\lambda}{2 \pi} \varphi
$$

In combination with the basic transformation,

$$
\begin{aligned}
\cos (\eta+\alpha) & =\cos \left(90^{\circ}-\theta+\alpha\right) \\
& =\cos \left[90^{\circ}-(\theta-\alpha)\right] \\
& =\sin (\theta-\alpha)
\end{aligned}
$$

$$
\sin (\theta-\alpha)=\frac{B^{2}+r^{2}-\left(r+\frac{\lambda}{2 \pi} \varphi\right)^{2}}{2 B r}
$$

In addition,

$$
\begin{gathered}
\cos \alpha=\frac{\sqrt{\mathrm{B}_{X}^{2}+\mathrm{B}_{Y}^{2}}}{B} \\
\sin \alpha=\frac{B_{\mathrm{Z}}}{B} \\
B=\sqrt{B_{X}^{2}+B_{\mathrm{Y}}^{2}+B_{Z}^{2}} \\
R_{H}=\sqrt{X_{1}^{2}+Y_{1}^{2}+Z_{1}^{2}}
\end{gathered}
$$

where $\varphi$ is the unwrapped phase of the ground point $\mathrm{P}$. $\left(B_{\mathrm{X}}, B_{\mathrm{Y}}\right.$, $\left.B_{\mathrm{Z}}\right)$ denotes three dimensional vector of the baseline. $\left(X_{1}, Y_{1}, Z_{1}\right)$ is the three dimensional coordinate of the antenna phase centre of the master image. $r$ is the slant range of the master image. These eight variables constitute the model observations for InSAR height measurement. The baseline is a significant variable for the model solution, thus the influence of the baseline error to height measurement was discussed comprehensively.

Based on the above theoretical derivation of the InSAR height measurement model, the error model of the baseline to height measurement can be obtained through the partial derivative processing. Assuming there is no coupling among the satellite orbit, slant range, interferometric phase and baseline, the relationship between the baseline error and height measurement can be derived as follows.

$$
\begin{gathered}
\sigma_{B_{X}}^{h}=\left|\frac{R_{H} \sin \theta}{R_{e}+h} \frac{B_{X}}{\mathrm{~B}_{\mathrm{Y}} \sin \theta+B_{Z} \cos \theta}\right| \sigma_{B_{X}} \\
\sigma_{B_{Y}}^{h}=\left|\frac{R_{H} \sin \theta}{R_{e}+h} \frac{B_{\mathrm{Y}}+r \cos \theta}{\mathrm{B}_{\mathrm{Y}} \sin \theta+B_{Z} \cos \theta}\right| \sigma_{B_{Y}} \\
\sigma_{B_{Z}}^{h}=\left|\frac{R_{H} \sin \theta}{R_{e}+h} \frac{B_{\mathrm{Z}}-r \sin \theta}{\mathrm{B}_{\mathrm{Y}} \sin \theta+B_{Z} \cos \theta}\right| \sigma_{B_{Z}}
\end{gathered}
$$

These formulas directly represent the error propagation characteristics from the baseline error to height measurement.

\subsection{Simulation strategy}

The simulation validation is the inverse process of the InSAR data processing. For the simulation validation, the main procedures consist of the DEM cropping, intensity simulation, flat phase and topographic phase simulation, as well as SLC generation.

Before the DEM cropping, the original DEM required to be mapped to the SAR coordinate space. The R-D model was employed to implement this procedure.

$$
\left\{\begin{array}{l}
1=\frac{X_{1}^{2}+Y_{1}^{2}}{\left(R_{e}+h\right)^{2}}+\frac{Z_{l}^{2}}{R_{p}^{2}} \\
r=\left|R_{s c}-R_{t c}\right| \\
f_{d}=-\frac{2}{\lambda} \frac{\left(R_{s c}-r\right) V_{s c}}{\left|R_{s c}-R_{t c}\right|}
\end{array}\right.
$$

Thus, the following representation can be obtained. 
where $r$ represents the slant range, $R_{s c}$ is the satellite position vector $\left(X_{1}, Y_{1}, Z_{1}\right), R_{t c}$ is the ground point position vector $\left(X_{\mathrm{t}}\right.$, $\left.Y_{\mathrm{t}}, Z_{\mathrm{t}}\right), f_{d}$ denotes the Doppler frequency shift, $\lambda$ is the radar wavelength, $V_{s c}$ is the satellite velocity vector $\left(X_{\mathrm{v}}, Y_{\mathrm{v}}, Z_{\mathrm{v}}\right), R_{e}$ is the earth radius, $R_{p}$ is the polar radius, and $h$ is the height of the target point.

For the intensity simulation, the radar incidence angle, heading angle and other parameters are required in the procedure. The relationship can be represented as follows.

$$
d_{(x, y)}=\sum_{(X, Y, Z)} I_{0} \frac{R_{\text {near }}^{4}}{R_{(X, Y, Z)}^{4}} \cos \theta_{(X, Y, Z)}
$$

where $I_{o}$ is the given microwave radiation intensity of the near range, $R_{\text {near }}$ is the near range delay, and $R_{(X, Y, Z)}$ is the slant range.

Generally, the phase of the master image does not require more treatment, which is given as a constant. Thus, the phase of the slave image can be derived in combination with the flat phase and topographic phase. The flat phase can be expressed as follows.

$$
\varphi_{f l t}=\varphi_{1}-\varphi_{2}=-\frac{2 \pi}{\lambda} \Delta r
$$

And the topographic phase can be represented as follows.

$$
\Delta \varphi=\frac{2 \pi B_{\perp} h}{\lambda r \sin \theta}
$$

When the amplitude $A$ and phase $\varphi$ were simulated, the complex value of the simulated master and slave image can be obtained.

where $a=A \cdot \cos \varphi, b=A \cdot \sin \varphi$.

$$
c=a+b i
$$

According to the above simulation strategy, the simulation validation of InSAR height measurement induced by baseline error can be implemented on the basis of TanDEM-X parameters and SRTM DEM.

\section{EXPERIMENTAL AREA AND DATA SET}

The study area located in the northeast of YuXian, China $\left(114.62^{\circ}-114.78^{\circ} \mathrm{E}, 39.88^{\circ}-39.99^{\circ} \mathrm{N}\right)$, shown as figure 2 . This area consists primarily of plain. The highest elevation is about $1050 \mathrm{~m}$, and the lowest elevation is about $800 \mathrm{~m}$.

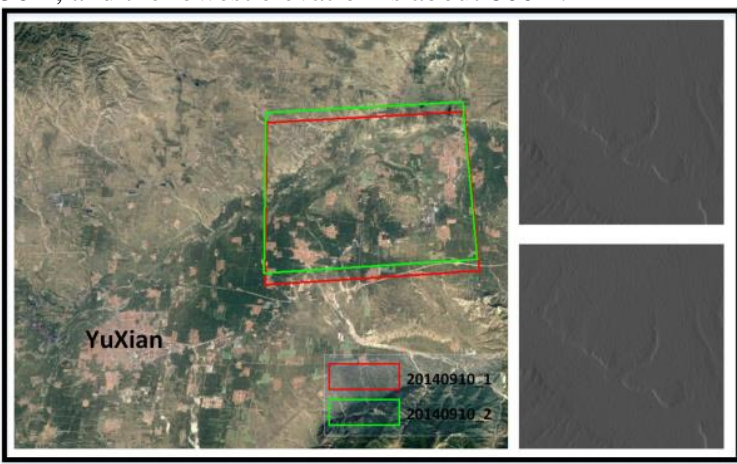

Figure 2. Study area and image range.
The TerraSAR-X/TanDEM-X data was acquired over the study area on 10 September 2014. The orbit direction of the data is ascending, and the incidence angle is about $36^{\circ}$. The azimuth and range resolution are $2.0 \mathrm{~m}$ and $1.4 \mathrm{~m}$, respectively. The experimental area covered a range of $10 \times 11 \mathrm{~km}^{2}$. The detailed information of TerraSAR-X/TanDEM-X data was listed in Table 1. Both of the simulation analysis and validation are implemented on the basis of the TerraSAR-X/TanDEM-X parameters.

\begin{tabular}{ccc}
\hline Parameters & $20140910 \_1$ & 20140910_2 \\
\hline Orbit inclination & $97.4472^{\circ}$ & $97.4472^{\circ}$ \\
Incidence angle & $36.0206^{\circ}$ & $36.0846^{\circ}$ \\
Azimuth resolution & 2.0225 & 2.0226 \\
Range resolution & 1.3641 & 1.3641 \\
Near slant range & 621147.7156 & 621113.6127 \\
Center slant range & 624557.2960 & 624523.1931 \\
Far slant range & 627966.8765 & 627932.7736 \\
PRF & \multicolumn{2}{c}{$3.49 \mathrm{KHz}$} \\
Wavelength & \multicolumn{2}{c}{$0.03125 \mathrm{~m}$} \\
Orbit altitude & \multicolumn{2}{c}{$514 \mathrm{KM}$} \\
Baseline & $-0.9588695 \mathrm{~m} \mathrm{256.2914386} \mathrm{m}$ \\
information & $-67.3983349 \mathrm{~m}$ \\
\hline Table 1. Detailed information of TerraSAR-X/TanDEM-X
\end{tabular}

According to the simulation strategy, the simulation of the SAR data over the study area was implemented in combination with the SRTM DEM and TanDEM-X parameters.

Firstly, the mapped and clipped DEM was obtained. Figure 3(a) shows the original SRTM DEM, and Figure 3(b) represents the mapped and clipped DEM.

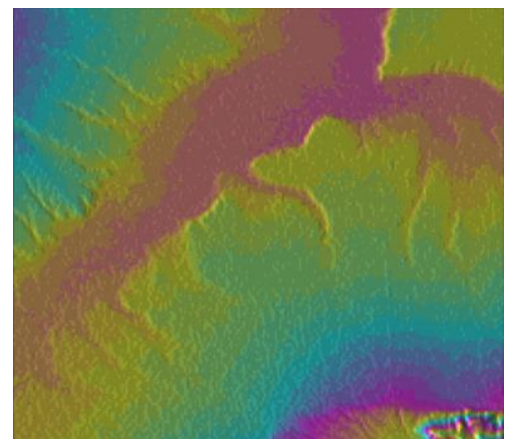

(a) Original DEM

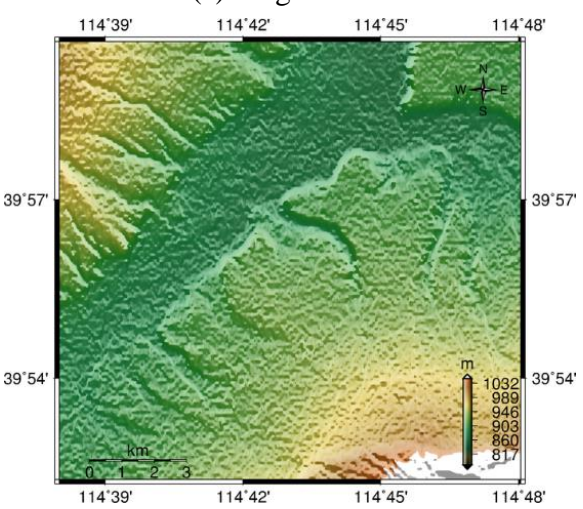

(b) Mapped and clipped DEM

Figure 3. SRTM DEM of the study area 
Secondly, the intensity image was simulated, shown as figure 4 . The simulated result shows comparable textural features with the SRTM DEM.
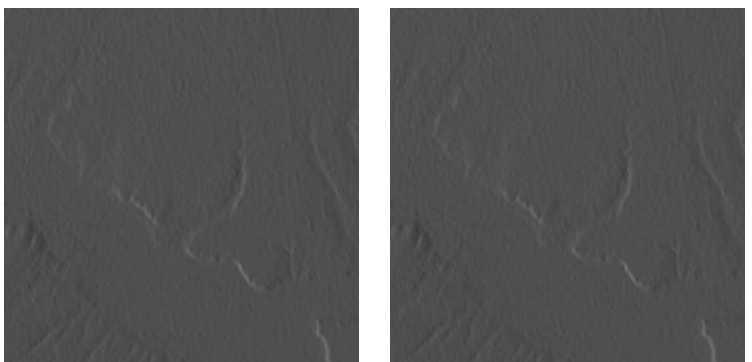

Figure 4. Simulated intensity image (Left: master image, Right: slave image)

Then, the phase simulation was performed. The simulated flat phase and topographic phase are shown as figure 5 .

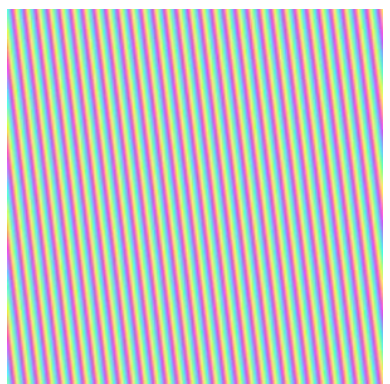

(a) Flat phase

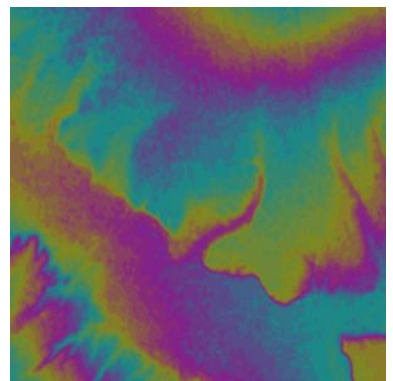

(b) Topographic phase
Figure 5. Simulated results of the flat phase and topographic phase.

At last, the simulated SAR image can be obtained in combination with the intensity and phase information.

\section{RESULTS AND DISCUSSIONS}

\subsection{Point scale evaluation based on the physical model}

According to the TanDEM-X parameters, the error of baseline vector changes from $1 \mathrm{~mm}$ to $10 \mathrm{~mm}$. The influence of the baseline error to height measurement was evaluated in combination with the error propagation formulas. Figure 6 shows the relationships between the baseline error and height error.

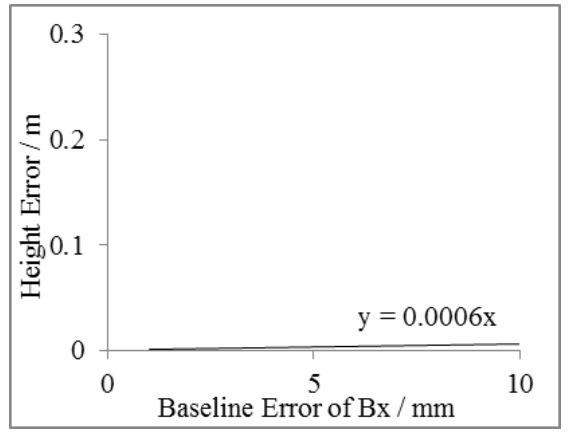

(a) Relationship between $B x$ error and height measurement

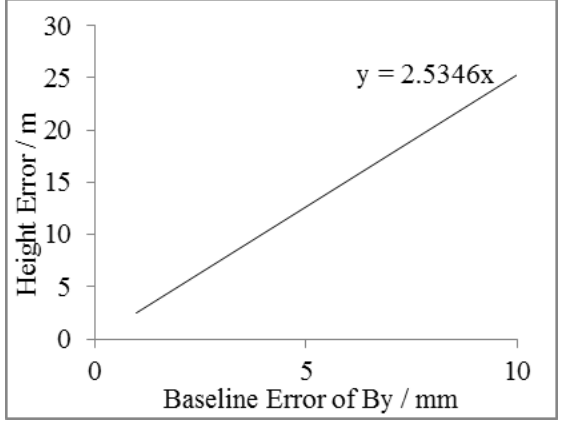

(b) Relationship between $B y$ error and height measurement

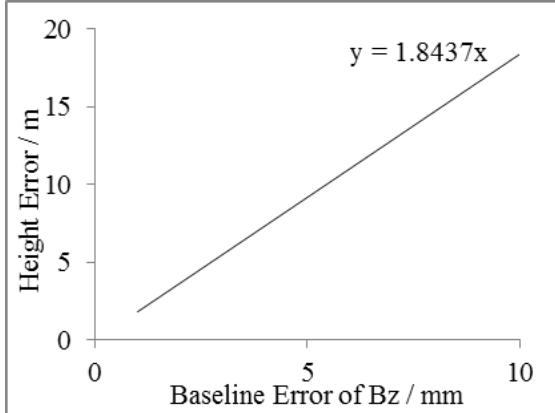

(c) Relationship between $\mathrm{Bz}$ error and height measurement

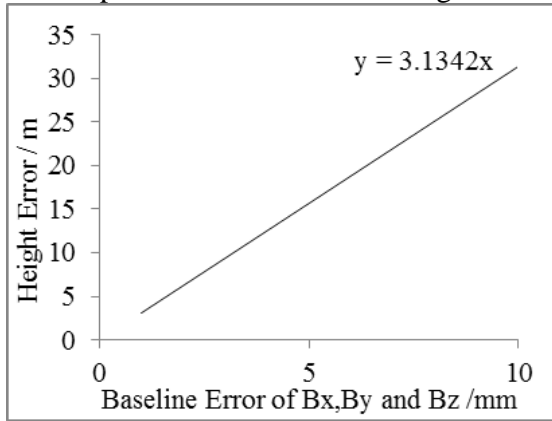

(d) Relationship between baseline vector error and height measurement

Figure 6. Relationships between the baseline error and height measurement

The simulation results indicated that with the increase of the baseline error the height measurement error linearly increases. The baseline component error of $B \mathrm{x}$ shows weak influence on height measurement, while the baseline component error of $B y$ and $B \mathrm{z}$ show significant influence on the height measurement. Several millimeters' error of the baseline can induce several meters' error for the height measurement. Therefore, the accurate measurement of the baseline vector is essential for InSAR height measurement.

\subsection{Spatial distribution validation of the baseline error to height measurement}

Based on the theoretical analysis of the influence of baseline error to height measurement, the spatial simulation validation of baseline error to height measurement was implemented in combination with the TanDEM-X parameters and SRTM DEM. The height error spatial distribution characteristics induced by baseline error was further quantitatively evaluated and discussed.

Firstly, given the different baseline vector error $\left(\Delta B_{\mathrm{X}}, \Delta B_{\mathrm{Y}}\right.$, $\Delta B_{\mathrm{Z}}$ ), the influence of baseline vector error to height measurement was quantitatively assessed. Based on the SRTM 
DEM, the height error distribution induced by the different baseline error was analysised and evaluated. Figure 7 shows the height error distribution graph corresponding to the different baseline error.
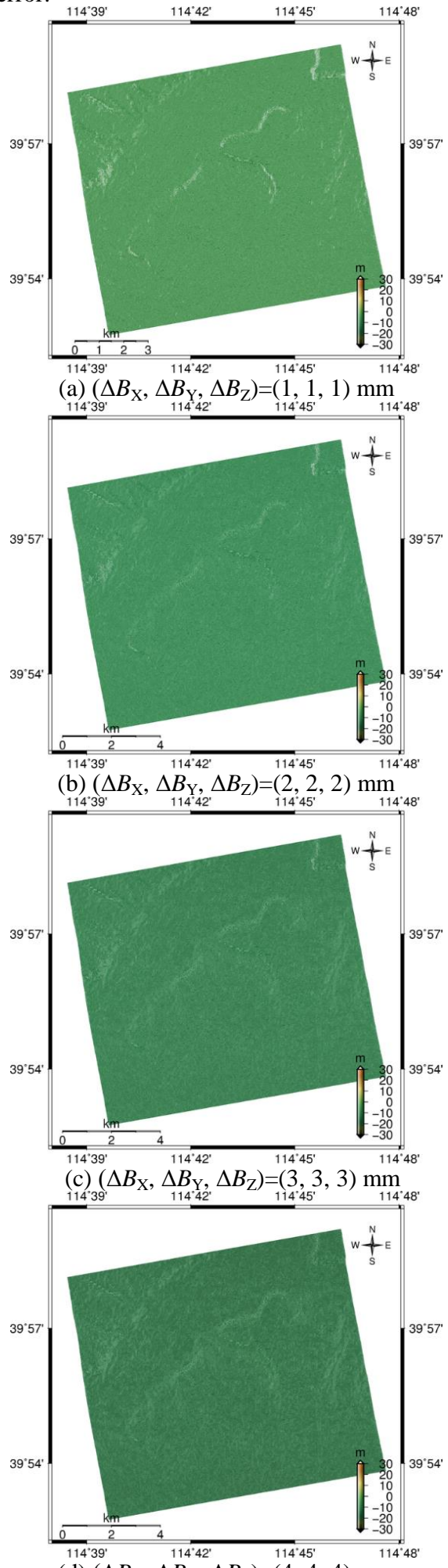

(d) $\left(\Delta B_{\mathrm{X}}, \Delta B_{\mathrm{Y}}, \Delta B_{\mathrm{Z}}\right)=(4,4,4) \mathrm{mm}$
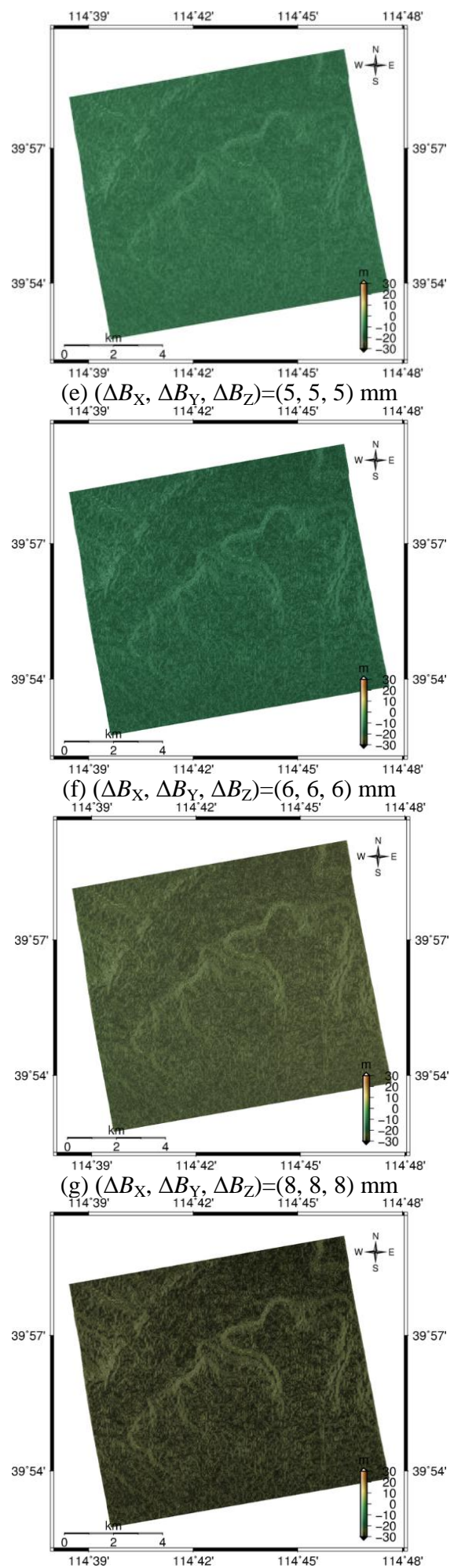

(h) $\left(\Delta B_{\mathrm{X}}, \Delta B_{\mathrm{Y}}, \Delta B_{\mathrm{Z}}\right)=(10,10,10) \mathrm{mm}$

Figure 7. Height error distribution graph induced by different baseline error

The simulation results indicate that the baseline error has significant influence on InSAR height measurement. With the increase of baseline error, the height measurement error increased gradually, which show good agreement with the theoretical analysis.

The spatial error distribution graph directly represented the pixel-to-pixel influence of baseline error to height measurement. 
Furthermore, the comprehensive influence of the baseline to height measurement was evaluated. The root mean square error (RMSE) was selected as the indicator. Table 2 shows the statistical results of the height measurement error induced by the different baseline error.

\begin{tabular}{cccccc}
\hline Baseline Error $/ \mathrm{mm}$ & 0 & 1 & 2 & 3 & 4 \\
\hline Height RMSE/m & 0.07 & 2.95 & 5.82 & 8.63 & 11.57 \\
\hline Baseline Error/mm & 5 & 6 & 8 & 10 \\
\hline Height RMSE/m & 14.44 & 17.31 & 23.06 & 28.80 \\
\hline Table 2. Statistical results of the height error induced by \\
baseline error
\end{tabular}

Figure 8 shows the relationship between the height measurement RMSE and baseline error.

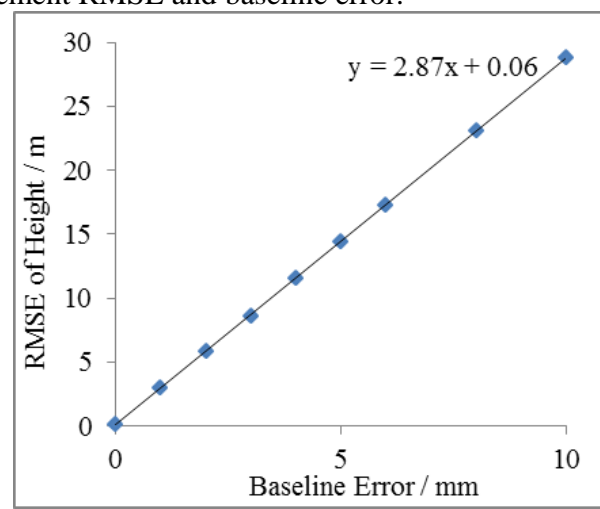

Figure 8. Relationship between the height RMSE and baseline error

The graph shows that the error propagation coefficient of the baseline error to height measurement can reach to 2.87 , which indicates the significant influence of the baseline error to height measurement. The simulation results show good agreement with the theoretical derivation, which also demonstrated the reliability of the InSAR height measurement error model. However, there is little difference between the theoretical analysis and simulation results. The following three aspects may explain the existence of the difference. Firstly, some assumption and approximation of the theoretical model may partially induce the deviation. For the theoretical derivation of baseline error model, the first derivative model was applied in this research. Actually, the higher derivative model may be more accurate for the theoretical derivation. Secondly, after the data simulation several processing steps are required to obtain the final DEM, especially for resampling, phase unwrapping and geocoding, which may introduce partial error to the DEM. Finally, for the theoretical derivation evaluation only the centre point of the image was applied. While for the simulation validation, the whole image was employed to validate the baseline error model. Therefore, the difference between the theoretical derivation and simulation validation exist in the experiments.

\section{CONCLUSIONS}

The theoretical derivation and simulation validation of the baseline error to InSAR height measurement were implemented in this research. According to point scale evaluation of the physical model and spatial distribution validation, the baseline error shows significant influence on the InSAR height measurement. Therefore, it is essential to obtain the accurate baseline vector for InSAR missions.

For future research on the novel SAR system, the accurate and reliable baseline acquirement is crucial. In addition, the validation for the baseline error model may be implemented on the basis of real SAR data. And higher derivative model for theoretical derivation may be promising in our further research.

\section{ACKNOWLEDGEMENTS}

This research was supported by the National Key R\&D Programme of China (No: 2017YFB0502700), Civilian Space Programme of China (No: D010102), National Basic Surveying, Mapping Science and Technology Plan (No: 2018KJ0204/ 2018KJ0304) and High-Resolution plan: The Demonstration System of High-Resolution Remote Sensing Application in Surveying and Mapping of China.

\section{REFERENCES}

Kroes, R., Montenbruck, O., Bertiger, W., and Visser, P. 2005. Precise GRACE baseline determination using GPS. GPS Solutions, 9(1), pp. 21-31.

Krieger, G., Moreira, A., Fiedler, H. et al. 2007. TanDEM-X: a satellite formation for high-resolution SAR interferometry. IEEE Transactions on Geoscience and Remote Sensing, 45(11), pp. 3317-3340.

Krieger, G. Hajnsek, I., Papathanassiou, K. P., Younis, M., and Moreira, A. 2010. Interferometric synthetic aperture radar (SAR) missions employing formation flying. Proceedings of the IEEE, 98(5), pp. 816-843.

Montenbruck, O., van Barneveld, P. W. L., Yoon, Y., and Visser, P. M. 2007. GPS-based precision baseline reconstruction for the TanDEM-X SAR-formation. in the 20th International Symposium on Space Flight Dynamics, pp. 24-28. Pitz, W., and Miller, D. 2010. The TerraSAR-X Satellite. IEEE Transactions on Geoscience and Remote Sensing, 48(2), pp. 615-622.

Wang, W. 2005. Optimal baseline design and error compensation for bistatic spaceborne InSAR. Proceedings of Fringe Workshop.

Werninghaus, R. and Buckreuss, S. 2010. The TerraSAR-X mission and system design. IEEE Transactions on Geoscience and Remote Sensing, 48(2), pp. 606-614.

Wermuth, M., Montenbruck, O., and Wendleder, A. 2011. Relative navigation for the TanDEM-X mission and evaluation with DEM calibration results. in the 22nd International Symposium on Space Flight Dynamics, Sao Jose dos Campos, Brazil.

Zink, M., Fiedler, H., Hajnsek, I., Krieger, G., Moreira, A., and Werner, M. 2006. The TanDEM-X mission concept. IEEE International Geoscience and Remote Sensing Symposium (IGARSS ’06), pp. 1938-1941. 